\title{
Article \\ Is Obesity a Risk or Protective Factor for Open-Angle Glaucoma in Adults? A Two-Database, Asian, Matched-Cohort Study
}

\author{
Wei-Dar Chen ${ }^{1,2} \oplus$, Li-Ju Lai ${ }^{1,2}$, Kang-Lung Lee ${ }^{3,4}$, Tzeng-Ji Chen ${ }^{4,5} \oplus$, Chia-Yen Liu ${ }^{6}$ and Yao-Hsu Yang ${ }^{6,7,8, *(\mathbb{})}$ \\ 1 Department of Ophthalmology, Chang Gung Memorial Hospital, Chiayi 33305, Taiwan; \\ weidar1023@gmail.com (W.-D.C.); lynnlai@cgmh.org.tw (L.-J.L.) \\ College of Medicine, Chang Gung University, Taoyuan 33302, Taiwan \\ 3 Department of Radiology, Taipei Veterans General Hospital, Taipei 11217, Taiwan; miguelkllee@gmail.com \\ 4 School of Medicine, National Yang Ming Chiao Tung University, Taipei 11221, Taiwan; tjchen@vghtpe.gov.tw \\ 5 Department of Family Medicine, Taipei Veterans General Hospital, Taipei 11217, Taiwan \\ 6 Health Information and Epidemiology Laboratory, Chang Gung Memorial Hospital, Chiayi 33305, Taiwan; \\ qchiayen@gmail.com \\ 7 Department of Traditional Chinese Medicine, Chang Gung Memorial Hospital, Chiayi 33305, Taiwan \\ 8 School of Traditional Chinese Medicine, College of Medicine, Chang Gung University, Taoyuan 33302, Taiwan \\ * Correspondence: r95841012@ntu.edu.tw; Tel.: +886-5-3621000 (ext. 3613)
}

\section{check for}

updates

Citation: Chen, W.-D.; Lai, L.-J.; Lee, K.-L.; Chen, T.-J.; Liu, C.-Y.; Yang,

Y.-H. Is Obesity a Risk or Protective

Factor for Open-Angle Glaucoma in

Adults? A Two-Database, Asian,

Matched-Cohort Study. J. Clin. Med. 2021, 10, 4021. https://doi.org/

$10.3390 / \mathrm{jcm} 10174021$

Academic Editors: Jose

Javier Garcia-Medina and Maria

Dolores Pinazo-Duran

Received: 7 August 2021

Accepted: 2 September 2021

Published: 6 September 2021

Publisher's Note: MDPI stays neutral with regard to jurisdictional claims in published maps and institutional affiliations.

Copyright: (c) 2021 by the authors. Licensee MDPI, Basel, Switzerland. This article is an open access article distributed under the terms and conditions of the Creative Commons Attribution (CC BY) license (https:/ / creativecommons.org/licenses/by/ $4.0 /)$.

\begin{abstract}
Obesity contributes to multiple systemic disorders; however, extensive discussion regarding obesity and open-angle glaucoma (OAG) remains limited, and conclusions in the existing literature diverge. This study aims to analyze the risk of OAG among obese adults in Taiwan. In this study, adults (aged $\geq 18$ years) with a diagnostic code of obesity or morbid obesity registered in the Longitudinal Health Insurance Database (LHID) 2000 and LHID2005 from 1 January 2001 to 31 December 2010 were included. All adults were traced until the diagnosis of OAG, the occurrence of death, or 31 December 2013. Risk of OAG was significantly higher in obese adults than in non-obese adults after multivariable adjustment (adjusted hazard ratio (aHR): 1.43 (95\% confidence interval (CI) 1.11-1.84)/aHR: 1.54 (95\% CI 1.23-1.94) in the LHID2000/LHID2005). Both databases demonstrated that young obese adults (aged $\leq 40$ years) had a remarkably increased risk of OAG compared with young non-obese adults (aHR 3.08 (95\% CI 1.82-5.21)/aHR 3.81 (95\% CI 2.26-6.42) in the LHID2000/LHID2005). This two-database matched-cohort study suggests that obese adults have an increased risk of OAG. In young adults, in particular, obesity could be a potential risk factor of OAG.
\end{abstract}

Keywords: obesity; open-angle glaucoma; risk factor; young adults

\section{Introduction}

Obesity represents one of the biggest health emergency issues worldwide. According to the World Health Organization, the prevalence of obesity (body mass index (BMI) $\geq 30 \mathrm{~kg} / \mathrm{m}^{2}$ ) in the United States was $36.2 \%$ in 2016 [1]; this figure was projected to soar to $50.7 \%$ by 2030 [2]. Similarly, the prevalence of obesity in Europe and Asia has increased exponentially over the past decade. Obesity has a multifactorial association with the environment, dietary habits, sedentary lifestyles, and genetics [3]. Obesity-derived metabolic dysregulation, inflammatory stress, and neural degeneration lead to a series of pathophysiological processes [4]. Numerous studies have indicated that obesity has a strong connection with diabetes mellitus, hypertension, ischemic heart disease, stroke, and Alzheimer's disease [5,6].

Although discussing the association between obesity and sight-threatening disorders has recently intensified [7], the relationship between obesity and open-angle glaucoma (OAG) remains controversial. OAG, the most prevalent subtype of glaucoma globally, is characterized by progressive damage of retinal ganglion cells (RGCs), enlarged optic disc cupping, and irreversible deterioration of the visual field. Lowering intraocular pressure 
(IOP) is considered to be the most effective strategy in preventing disease progression, although the etiology of OAG has been not completely elucidated.

To date, several studies have reported that obesity has a positive correlation with increased IOP [8-10]. Nevertheless, whether obesity is a risk factor for OAG remains inconclusive $[11,12]$. In the Rotterdam study, there was no significant association between BMI and OAG after multivariable adjustment [13]. However, Newman-Casey showed that obese patients had a $14 \%$ increased risk of OAG in the univariable analysis, and obese women had a $6 \%$ increased hazard for OAG after multivariable adjustment [14]. Conversely, Pasquale reported that increased BMI was associated with a $6 \%$ lower risk of OAG in Caucasian women [15]. Additionally, Kim et al. showed that overweight status is a protective factor of OAG [16]. Prompted by inconsistent reports of the association between obesity and OAG, the present 13-year matched-cohort study aimed to analyze the risk of OAG among obese individuals using data from two Taiwanese population-based longitudinal databases.

\section{Materials and Methods}

\subsection{Databases}

In this study, participant data were collected from the Longitudinal Health Insurance Database (LHID) 2000 and LHID2005, which belong to the National Health Insurance research database (NHIRD) in Taiwan. The National Health Insurance program is a universal and compulsory health insurance program benefiting $>99.6 \%$ of the Taiwanese population to date, and data of the NHIRD, including registration files and original claim data, have been used in many studies with high academic value. The LHID2000 contains $1,000,000$ de-identified insurance claim data randomly retrieved from the year 2000 Registry of Beneficiaries of the NHIRD (23.75 million) during the period of 1 January 2000 to 31 December 2000 by Oracle's internal random number generator. Likewise, the LHID2005 encompasses 1,000,000 insurance claim data drawn from the year 2005 Registry of Beneficiaries of the NHIRD (25.68 million) during the period of 1 January 2005 to 31 December 2005 following the same method. Through statistic validation, there was no selection bias on age, sex, or insurance premiums between the LHID and the NHIRD. Both the LHID2000 and LHID2005 adopted the 2001 Edition of International Classification of Diseases, Ninth Revision, Clinical Modification (ICD-9-CM). This study was approved by the Institutional Review Board (IRB) of Chang Gung Medical Foundation, and adhered to the tenets of the Declaration of Helsinki. Given the retrospective nature of this study and the use of de-identified patient data in the LHID, the IRB of the Chang Gung Medical foundation approved that the requirements of informed consent were waived.

\subsection{Study Design}

A flowchart of enrollment of obese and non-obese groups is presented in Figure 1. The LHID2000 and LHID2005 were separately reviewed in this matched-cohort study. There were 17,256 and 17,474 individuals aged $\geq 18$ years included from the LHID2000 and LHID2005. The obese individuals were qualified with the coding of obesity or morbid obesity (ICD-9-CM codes 278.0, 278.00, 278.01) between 1 January 1997 and 31 December 2013. We washed out the initial 4-year period to eliminate individuals with incomplete data and those diagnosed with obesity before 2001 (LHID2000/LHID2005: 1889/1880 individuals). Simultaneously, individuals with a new diagnosis of obesity after 2010 were excluded to confirm that individuals can be traced for at least 3 years (LHID2000/LHID2005: $3365 / 3397$ individuals). Further, individuals diagnosed with OAG before a coding of obesity were also excluded (LHID2000/LHID2005: 60/74 individuals). Final numbers of 11,939 and 12,118 individuals with a new diagnosis of obesity between 2001 and 2010 were recruited in obese groups of the LHID2000 and LHID2005. Individuals without a diagnosis of overweight or obesity (ICD-9-CM codes: 278.0, 278.00, 278.01, 278.02, and 278.1) were included in the non-obese group. The obese individuals were matched with the non-obese individuals at a 1:4 ratio by sex, age, urbanized level, and income. The stratification of 
urbanization level and income followed the criteria reported in a previous LHID study [17]. All individuals were traced from the index date to the diagnosis of OAG, occurrence of death, or 31 December 2013.

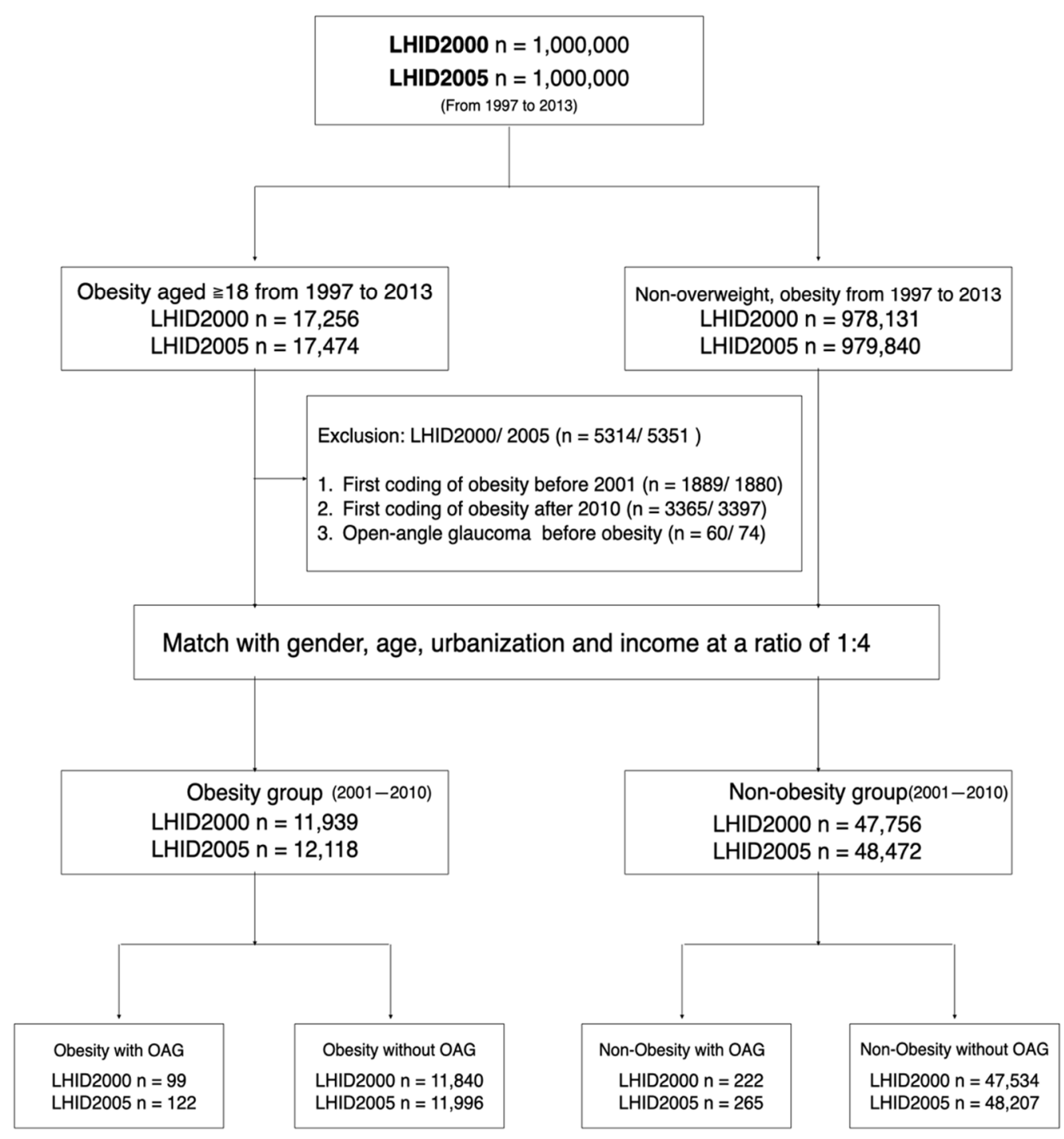

Figure 1. Flowchart of enrollment and allocation of adults with obesity and non-obesity in a two-database matched-cohort study. Obese adults aged $\geq 18$ years in the LHID2000 and LHID2005 between 1 January 1997 and 31 December 2013 were included. Adults first diagnosed with obesity before 2001, adults first diagnosed with obesity after 2010, and adults diagnosed with OAG before obesity were excluded in this study. Obese adults were matched with non-obese adults at a 1:4 ratio by sex, age, urbanization level, and income. All adults were traced until the death, occurrence of OAG, or 31 December 2013. LHID = longitudinal health insurance database; OAG = open-angle glaucoma.

To increase the validation of OAG, outcomes were rigorously defined as the coding of OAG (ICD-9-CM codes 365.1, 365.10, 365.11, 365.12), with a treatment involving antiglaucoma drugs or surgeries $\geq 2$ times, adjudicated by an ophthalmologist(s) over one year. Multiple covariates were collected for adjustment of the risk of OAG: diabetes mellitus (ICD-9-CM codes 250x), hypertension (ICD-9-CM codes 401x-405x), hyperlipidemia (ICD9-CM codes 272x), ischemic heart disease (IHD) (ICD-9-CM codes 410x-414x), chronic kidney disease (CKD) (ICD-9-CM codes 585x and 586x), myopia (ICD-9-CM codes 367.1), migraine (ICD-9-CM codes 346x), hypothyroidism (ICD-9-CM code 244.9), obstructive sleep apnea (OSA) (ICD-9-CM codes 327.23, 780.51, 780.53, and 780.57), and hypotension (ICD-9- 
$\mathrm{CM}$ codes $458 \mathrm{x})$. The inclusion criteria for covariates were diagnostic coding $\geq 1$ time in admission or $\geq 3$ times in ambulatory visits.

\subsection{Statistical Analyses}

All statistical data in this study were processed and analyzed using SAS version 9.4 (SAS Inc., Cary, NC, USA). For baseline characteristics, a chi-squared test and Student's $t$ test were used to analyze categorical and continuous variables, respectively. The cumulative incidence of OAG was calculated using the Kaplan-Meier method and a log-rank test. A multivariable Cox proportional regression was applied to estimate the adjusted hazard ratio (aHR) and 95\% confidence interval (CI) for risk of OAG. The main model for adjustment consisted of demographic variables: sex, age, urbanization level, and income, and five cardinal covariates associated with risk of OAG: diabetes mellitus, hypertension, hyperlipidemia, ischemic heart disease, and chronic kidney disease. Another five potential covariates of OAG-myopia, hypothyroidism, migraine, obstructive sleep apnea, and hypotension-were added one by one into the main model for testing the stability of the sensitivity analysis. Ultimately, sex, age, and covariates were tested for risk of OAG in multivariable stratified analysis. Statistical significance was defined as a two-sided $p<0.05$.

\section{Results}

In the LHID2000, OAG occurrence was 99 in 11,939 obese individuals, and the incidence rate of OAG was 1.0 and 0.6 per 1000 person-years in the obese and non-obese groups (incidence rate ratio (IRR) 1.79), respectively. In the LHID2005, OAG occurrence was 122 in 12,118 obese individuals, and the incidence rate of OAG was 1.3 and 0.7 per 1000 personyears in the obese and non-obese groups (IRR 1.85), respectively. The average follow-up was $7.91 \pm 2.93 / 7.93 \pm 2.93$ years in the obese/non-obese groups of the LHID2000, respectively, and $7.88 \pm 2.92 / 7.89 \pm 2.92$ years in the obese/non-obese groups of LHID2005, respectively. The ratio of females to males was 2:1 in both databases. For age distribution, the ratio of obese individuals aged $\leq 40$ and $>40$ years was approximately 1:1 in both databases. Obese adults had a higher rate and frequency of ophthalmology visits than non-obese adults. Additionally, obese adults had higher rates of diabetes mellitus, hypertension, hyperlipidemia, ischemic heart disease, chronic kidney disease, myopia, obstructive sleep apnea, migraine, and hypothyroidism than non-obese adults in both databases (Table 1).

Table 1. Baseline characteristics with incidence of open-angle glaucoma in obese and non-obese adults.

\begin{tabular}{|c|c|c|c|c|c|c|}
\hline & \multicolumn{3}{|c|}{ LHID2000 } & \multicolumn{3}{|c|}{ LHID2005 } \\
\hline & Obesity & Non-Obesity & & Obesity & Non-Obesity & \\
\hline Variables & $n(\%)$ & $n(\%)$ & $p$-value & $n(\%)$ & $n(\%)$ & $p$-value \\
\hline Total & 11,939 & 47,756 & & 12,118 & 48,472 & \\
\hline Gender & & & 1.00 & & & 1.00 \\
\hline Female & $7718(64.6)$ & $30,872(64.6)$ & & 7815 (64.5) & $31,260(64.5)$ & \\
\hline Male & $4221(35.4)$ & $16,884(35.4)$ & & $4303(35.5)$ & $17,212(35.5)$ & \\
\hline Age & & & 1.00 & & & 1.00 \\
\hline $18-30$ & 3135 (26.3) & $12,540(26.3)$ & & $3098(25.6)$ & $12,392(25.6)$ & \\
\hline $31-40$ & $2858(23.9)$ & $11,432(23.9)$ & & $2961(24.4)$ & $11,844(24.4)$ & \\
\hline $41-50$ & $2660(22.3)$ & $10,640(22.3)$ & & $2645(21.8)$ & $10,580(21.8)$ & \\
\hline $51-60$ & 1992 (16.7) & 7968 (16.7) & & $2054(17.0)$ & $8216(17.0)$ & \\
\hline $61-70$ & $886(7.4)$ & $3544(7.4)$ & & $944(7.8)$ & $3776(7.8)$ & \\
\hline$>70$ & $408(3.4)$ & $1632(3.4)$ & & $416(3.4)$ & $1664(3.4)$ & \\
\hline Urbanization level & & & 1.00 & & & 1.00 \\
\hline 1(City) & 3965 (33.2) & $15,860(33.2)$ & & 3765 (31.1) & $15,060(31.1)$ & \\
\hline 2 & 5477 (45.9) & $21,908(45.9)$ & & $5837(48.2)$ & $23,348(48.2)$ & \\
\hline 3 & $1673(14.0)$ & $6692(14.0)$ & & $1746(14.4)$ & $6984(14.4)$ & \\
\hline 4(Villages) & $824(6.9)$ & $3296(6.9)$ & & $770(6.4)$ & $3080(6.4)$ & \\
\hline
\end{tabular}


Table 1. Cont.

\begin{tabular}{|c|c|c|c|c|c|c|}
\hline & \multicolumn{3}{|c|}{ LHID2000 } & \multicolumn{3}{|c|}{ LHID2005 } \\
\hline & Obesity & Non-Obesity & & Obesity & Non-Obesity & \\
\hline Income & & & 1.00 & & & 1.00 \\
\hline 0 & $3952(33.1)$ & $15,808(33.1)$ & & $2447(20.2)$ & $9788(20.2)$ & \\
\hline $1-15,840$ & $1883(15.8)$ & $7532(15.8)$ & & $1811(14.9)$ & 7244 (14.9) & \\
\hline $15,841-25,000$ & $3990(33.4)$ & $15,960(33.4)$ & & $3742(30.9)$ & $14,968(30.9)$ & \\
\hline$\geq 25,001$ & $2114(17.7)$ & $8456(17.7)$ & & $4118(34.0)$ & $16,472(34.0)$ & \\
\hline \multirow{2}{*}{$\begin{array}{c}\text { OPH visit } \\
\text { Frequency of } \mathrm{OPH} \\
\text { visit }\end{array}$} & $3856(32.3)$ & $12,082(25.3)$ & $<0.001$ & $3995(33.0)$ & $12,731(26.3)$ & $<0.001$ \\
\hline & & & $<0.001$ & & & $<0.001$ \\
\hline 0 & $8083(67.7)$ & $35,674(74.7)$ & & $8123(67.0)$ & $35,741(73.7)$ & \\
\hline $1-2$ & $2458(20.6)$ & 8061 (16.9) & & $2564(21.2)$ & $8470(17.5)$ & \\
\hline$>2$ & 1398 (11.7) & $4021(8.4)$ & & $1431(11.8)$ & $4261(8.8)$ & \\
\hline \multicolumn{7}{|l|}{ Comorbidity } \\
\hline Diabetes mellitus & $3494(29.3)$ & $5419(11.4)$ & $<0.001$ & $3642(30.1)$ & $5810(12.0)$ & $<0.001$ \\
\hline Hypertension & $5576(46.7)$ & $10,818(22.7)$ & $<0.001$ & $5571(46.0)$ & $11,434(23.6)$ & $<0.001$ \\
\hline Hyperlipidemia & $5202(43.6)$ & $8326(17.4)$ & $<0.001$ & $5353(44.2)$ & $8939(18.4)$ & $<0.001$ \\
\hline IHD & 2275 (19.1) & $4510(9.4)$ & $<0.001$ & $2306(19.0)$ & $4867(10.0)$ & $<0.001$ \\
\hline CKD & $381(3.2)$ & $969(2.0)$ & $<0.001$ & $372(3.1)$ & $1027(2.1)$ & $<0.001$ \\
\hline Myopia & $1169(9.8)$ & $3422(7.2)$ & $<0.001$ & $1207(10.0)$ & $3562(7.4)$ & $<0.001$ \\
\hline Migraine & $697(5.8)$ & $1618(3.4)$ & $<0.001$ & $730(6.0)$ & $1815(3.7)$ & $<0.001$ \\
\hline Hypothyroidism & $269(2.3)$ & $354(0.7)$ & $<0.001$ & $266(2.2)$ & $404(0.8)$ & $<0.001$ \\
\hline OSA & $463(3.9)$ & $319(0.7)$ & $<0.001$ & $428(3.5)$ & $313(0.7)$ & $<0.001$ \\
\hline Hypotension & $72(0.6)$ & $225(0.5)$ & 0.067 & $71(0.6)$ & $234(0.5)$ & 0.151 \\
\hline No. of OAG & 99 & 222 & & 122 & 265 & \\
\hline Incidence \% & 1.0 & 0.6 & $<0.001$ & 1.3 & 0.7 & $<0.001$ \\
\hline IRR (95\% CI) & \multicolumn{2}{|c|}{$1.79(1.41-2.27)$} & \multicolumn{4}{|c|}{$1.85(1.49-2.29)$} \\
\hline
\end{tabular}

$\mathrm{CI}=$ confidence interval; $\mathrm{CKD}=$ chronic kidney disease; $\mathrm{IHD}=$ ischemic heart disease; $\mathrm{LHID}=$ Longitudinal Health Insurance Database $\mathrm{OAG}=$ open-angle glaucoma; OSA = obstructive sleep apnea; $\mathrm{OPH}=$ ophthalmology $\mathrm{IRR}$ = incidence rate ratio.

Kaplan-Meier curve analysis revealed that the obese group had a significantly higher cumulative incidence of OAG than the non-obese group in both databases $(p<0.001)$ (Figure 2A,B). In the age-stratified analysis, both the young and older obese groups still showed an increased accumulation of OAG compared with the young and older non-obese groups, and the young obese group displayed a remarkable accumulation of OAG in both databases (Figure 2C,D).

The risk of OAG in the multivariable Cox proportional hazard regression are summarized in Table 2. The risk of OAG occurrence was significantly higher in the obese group than in the non-obese group after adjusting for the main model (aHR 1.43 in the LHID2000; aHR 1.54 in the LHID2005). Men appeared to have a higher risk of OAG than women; however, the difference did not reach statistical significance. Older adults (aged $>40$ years) had an increased risk of OAG compared with young adults (aged $\leq 40$ years). Among covariates, diabetes mellitus was a prominent risk factor for OAG in both databases. 
A

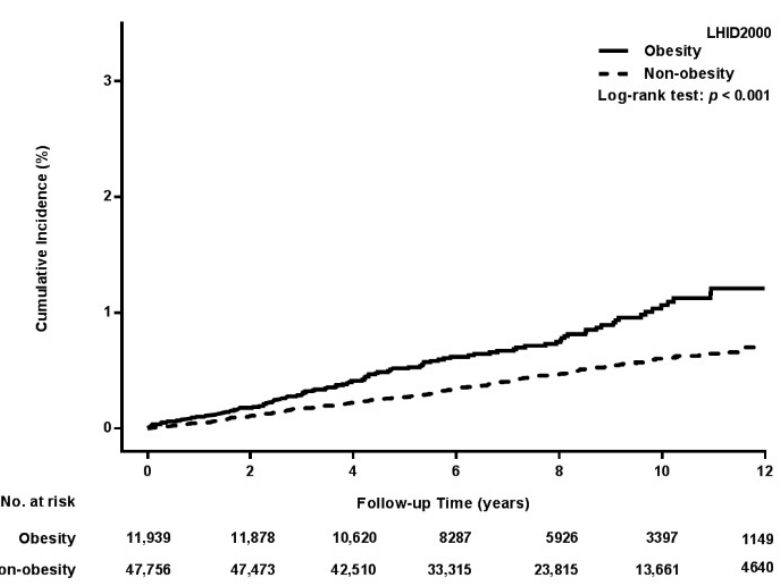

B

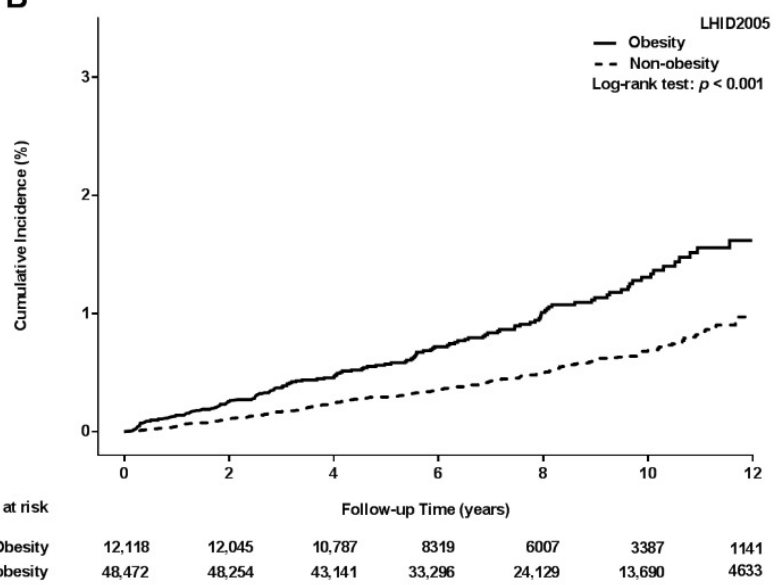

C

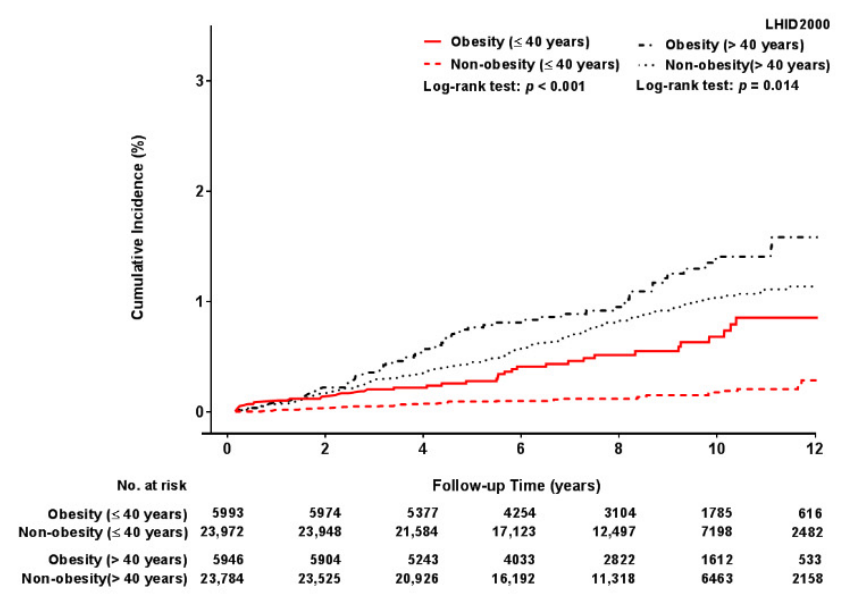

D

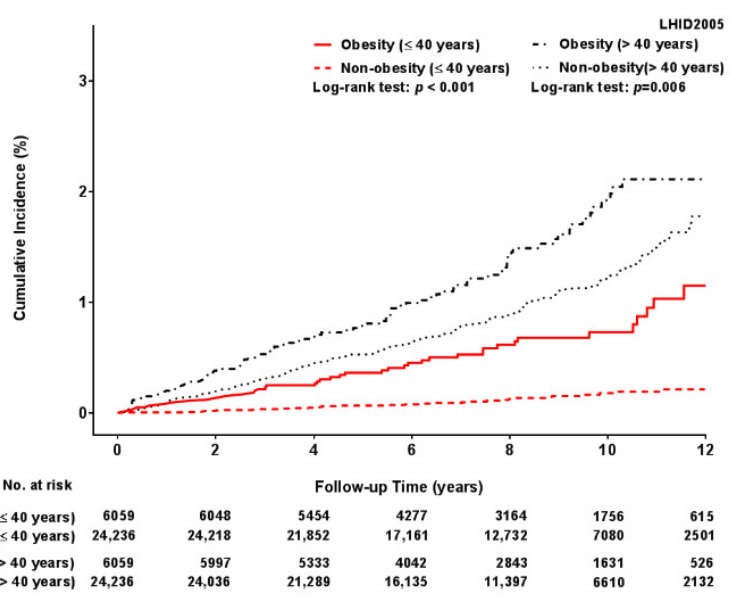

Figure 2. Cumulative incidence of open-angle glaucoma in obese and non-obese adults. (A) Cumulative incidence of OAG in the LHID2000; (B) cumulative incidence of OAG in the LHID2005; (C) age-stratified analysis for the cumulative incidence of OAG in the LHID2000; (D) age-stratified analysis for the cumulative incidence of OAG in the LHID2005. Both the LHID2000 and LHID2005 demonstrated that the cumulative incidence of OAG was higher in obese adults than non-obese adults (Log-rank test $p<0.001$ ). In the age-stratified analysis, young obese adults (red line) displayed a remarkable cumulative incidence of OAG compared with young non-obese adults (red dotted line) (Log-rank test $p<0.001$ ) although both the young and older obese adults had a significantly higher cumulative incidence of OAG. LHID = longitudinal health insurance database; $\mathrm{OAG}=$ open-angle glaucoma.

Table 2. Multivariable cox proportional hazard regression of the association between open-angle glaucoma and potential risk factors.

\begin{tabular}{|c|c|c|c|c|c|c|c|c|}
\hline \multirow[b]{2}{*}{ Variables } & \multicolumn{4}{|c|}{ LHID2000 } & \multicolumn{4}{|c|}{ LHID2005 } \\
\hline & $\begin{array}{c}\text { Crude HR } \\
(95 \% \text { CI) }\end{array}$ & $p$-Value & $\begin{array}{c}\text { aHR } \\
(95 \% \mathrm{CI})\end{array}$ & $p$-Value & $\begin{array}{c}\text { Crude HR } \\
(95 \% \text { CI })\end{array}$ & $p$-Value & $\begin{array}{c}\text { aHR } \\
(95 \% \mathrm{CI})\end{array}$ & $p$-Value \\
\hline \multicolumn{9}{|l|}{ Exposure } \\
\hline Non-obesity & 1 (Reference) & & 1 (Reference) & & 1 (Reference) & & 1 (Reference) & \\
\hline Obesity & $1.79(1.41-2.27)$ & $<0.001$ & $1.43(1.11-1.84)$ & 0.006 & $1.85(1.49-2.29)$ & 122 & $1.54(1.23-1.94)$ & $<0.001$ \\
\hline \multicolumn{9}{|l|}{ Gender } \\
\hline Female & 1 (Reference) & & 1 (Reference) & & 1 (Reference) & & 1 (Reference) & \\
\hline Male & $1.23(0.98-1.54)$ & 0.076 & $1.21(0.95-1.52)$ & 0.120 & $1.16(0.95-1.43)$ & 0.153 & $1.19(0.96-1.47)$ & 0.109 \\
\hline
\end{tabular}


Table 2. Cont.

\begin{tabular}{|c|c|c|c|c|c|c|c|c|}
\hline \multirow[b]{2}{*}{ Variables } & \multicolumn{4}{|c|}{ LHID2000 } & \multicolumn{4}{|c|}{ LHID2005 } \\
\hline & $\begin{array}{c}\text { Crude HR } \\
\text { (95\% CI) }\end{array}$ & $p$-Value & $\begin{array}{c}\text { aHR } \\
(95 \% \mathrm{CI})\end{array}$ & $p$-Value & $\begin{array}{c}\text { Crude HR } \\
\text { (95\% CI) }\end{array}$ & $p$-Value & $\begin{array}{c}\text { aHR } \\
(95 \% \mathrm{CI})\end{array}$ & $p$-Value \\
\hline Age $\leq 40$ & 1 (Reference) & & 1 (Reference) & & 1 (Reference) & & 1 (Reference) & \\
\hline Age $>40$ & $3.83(2.94-5.00)$ & $<0.001$ & $3.09(2.26-4.25)$ & $<0.001$ & $4.75(3.66-6.15)$ & $<0.001$ & $3.52(2.64-4.70)$ & $<0.001$ \\
\hline \multicolumn{9}{|l|}{ Comorbidity } \\
\hline \multicolumn{9}{|l|}{ Diabetes } \\
\hline No & 1 (Reference) & & 1 (Reference) & & 1 (Reference) & & 1 (Reference) & \\
\hline Yes & $2.91(2.31-3.66)$ & $<0.001$ & $1.55(1.18-2.04)$ & 0.002 & $3.04(2.47-3.73)$ & $<0.001$ & $1.61(1.26-2.06)$ & $<0.001$ \\
\hline \multicolumn{9}{|c|}{ Hypertension } \\
\hline No & 1 (Reference) & & 1 (Reference) & & 1 (Reference) & & 1 (Reference) & \\
\hline Yes & $2.68(2.16-3.34)$ & $<0.001$ & $1.22(0.92-1.61)$ & 0.170 & $3.00(2.46-3.67)$ & $<0.001$ & $1.38(1.07-1.77)$ & 0.012 \\
\hline \multicolumn{9}{|c|}{ Hyperlipidemia } \\
\hline No & 1 (Reference) & & 1 (Reference) & & 1 (Reference) & & 1 (Reference) & \\
\hline Yes & $2.51(2.01-3.12)$ & $<0.001$ & $1.14(0.87-1.50)$ & 0.347 & $2.27(1.86-2.78)$ & $<0.001$ & $0.90(0.71-1.15)$ & 0.412 \\
\hline \multicolumn{9}{|l|}{ IHD } \\
\hline No & 1 (Reference) & & 1 (Reference) & & 1 (Reference) & & 1 (Reference) & \\
\hline Yes & $2.66(2.08-3.42)$ & $<0.001$ & $1.27(0.95-1.68)$ & 0.106 & $2.60(2.07-3.25)$ & $<0.001$ & $(1.170 .91-1.50)$ & 0.231 \\
\hline \multicolumn{9}{|l|}{ CKD } \\
\hline No & 1 (Reference) & & 1 (Reference) & & 1 (Reference) & & 1 (Reference) & \\
\hline Yes & $2.17(1.29-3.64)$ & 0.003 & $1.02(0.60-1.74)$ & 0.934 & $2.05(1.28-3.30)$ & 0.003 & $0.93(0.57-1.51)$ & 0.764 \\
\hline
\end{tabular}

$\mathrm{CKD}=$ chronic kidney disease; IHD = ischemic heart disease; IRR = incidence rate ratio; LHID = Longitudinal Health Insurance Database OAG = open-angle glaucoma .

In sensitivity analysis of the risk of OAG (Figure 3), after adjustment for full model or potential covariates, the aHR of OAG occurrence in obesity remained steady, from 1.32 to 1.43 in the LHID2000, and from 1.46 to 1.58 in the LHID2005.

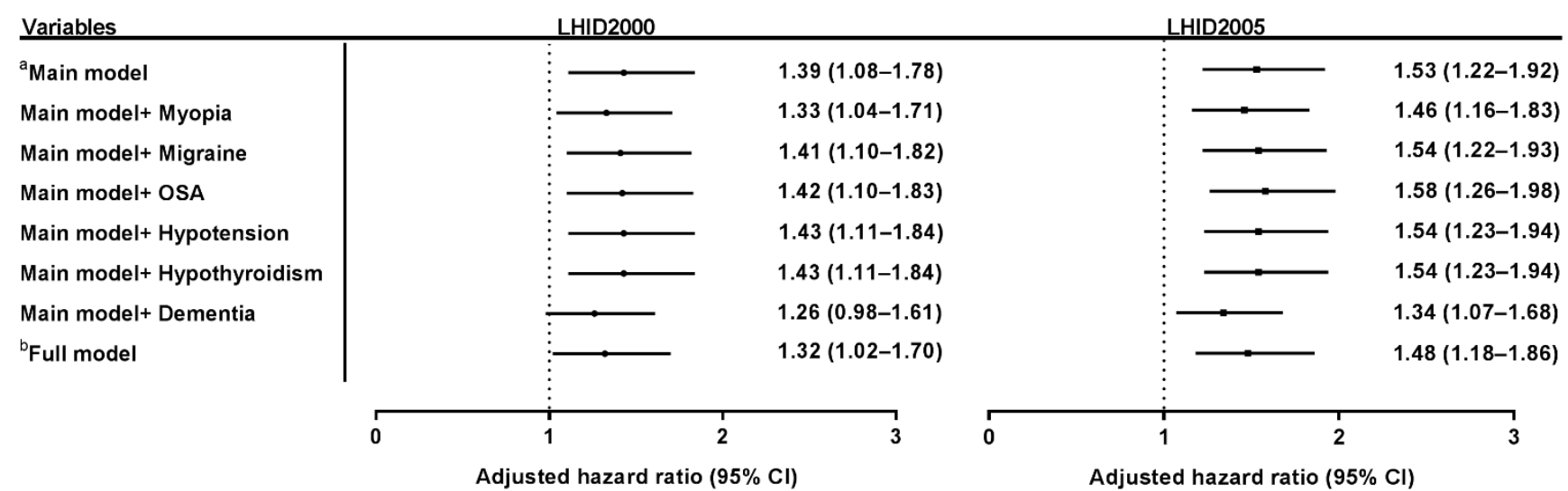

Figure 3. Sensitivity analysis of the risk of open-angle glaucoma in obesity. Sensitivity analysis in both the LHID2000 and LHID2005 showed that the adjusted hazard ratio of OAG remained stable after being adjusted according to the full model and main model with each potential covariate (myopia, migraine, OSA, hypotension, and hypothyroidism). The adjusted hazard ratio of OAG was 1.32 to 1.43 in the LHID2000, and 1.49 to 1.58 in the LHID2005. ${ }^{a}$ Main model adjusted for age, gender, income, urbanization, diabetes mellitus, hypertension, hyperlipidemia, ischemic heart disease, and chronic kidney disease ${ }^{b}$ Full model adjusted for the main model, myopia, hypothyroidism, migraine, OSA, and hypotension. OAG = open-angle glaucoma; OSA = obstructive sleep apnea. 
The multivariable stratified analysis is presented in Figure 4. In the age-stratified analysis, there was a demonstrably higher risk in young obese adults than in young nonobese adults, but there was no statistical significance of OAG risk in older adults. In the sex-stratified analysis, obese men had a higher risk of OAG compared with the non-obese men in both databases; however, obese women had an unremarkable (LHID2000) and borderline (LHID2005) risk of OAG. Moreover, adults with at least one ophthalmology visit were analyzed, and the result showed that obese adults still had a higher risk of OAG than non-obese adults in both databases. In the covariate-stratified analysis, the results revealed that in the LHID2005, obese adults without each covariate had a significantly higher risk of OAG than non-obese adults without each covariate, but obese adults with each covariate had a nonsignificant hazard for OAG compared with non-obese adults with each covariate. There was a similar tendency in most analyses of covariates in the LHID2000, although the analysis of hypertension and hyperlipidemia was discordant between the LHID2000 and LHID2005.

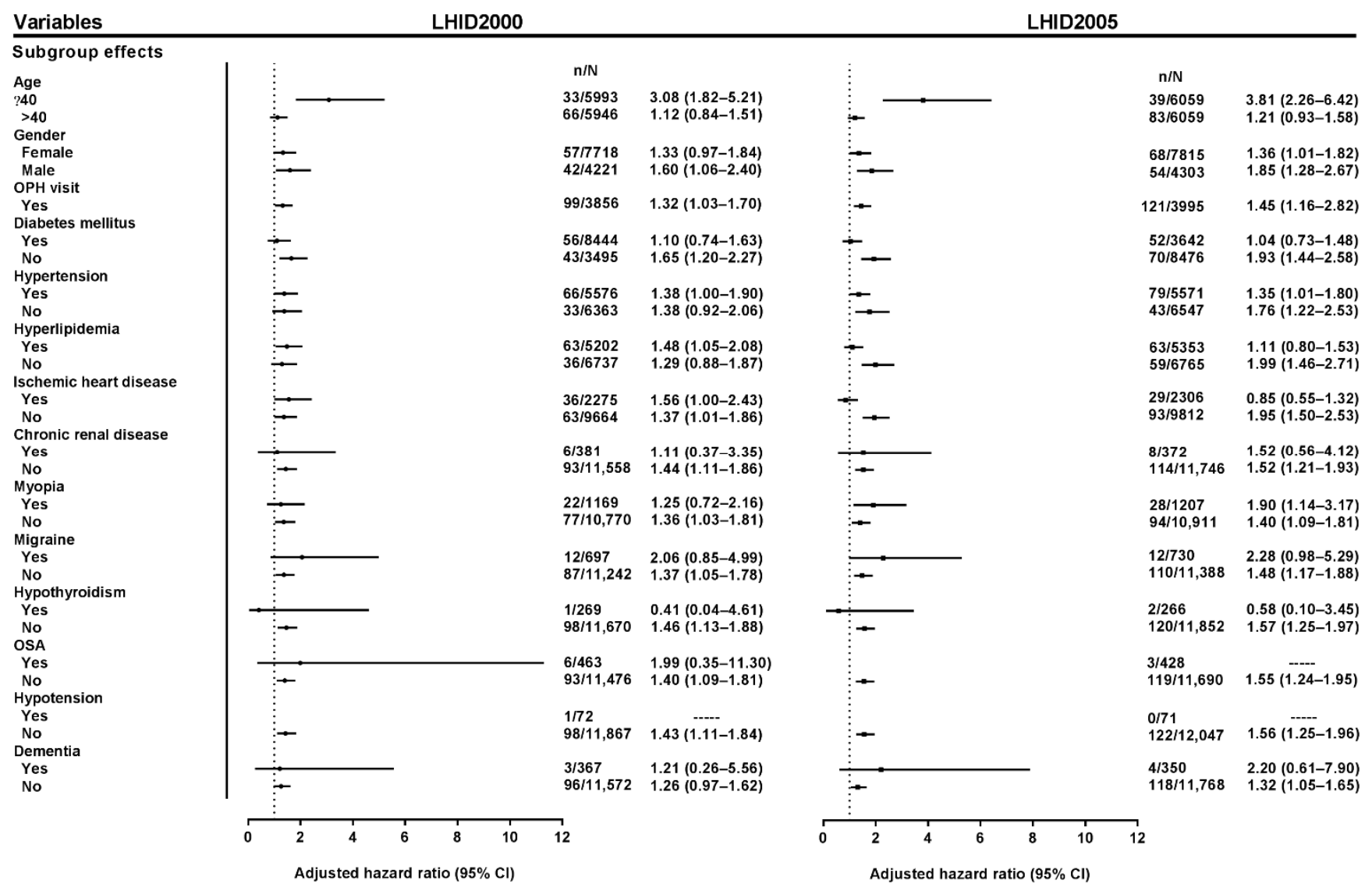

Figure 4. Multivariable stratified hazard analysis of the association between obesity and open-angle glaucoma. All adjusted hazard ratios in the multivariable stratified analysis were adjusted by the main model. The results in both the LHID2000 and LHID2005 displayed that obesity was a remarkable hazard of OAG in young (aged $\leq 40$ years) obese adults compared with young non-obese adults. In gender stratification, the risk of OAG was more significant in obese men than non-obese men. As for the stratified analysis of covariates, in the LHID2005, the obese group without each covariate had an increased risk of OAG compared with the non-obese group without each covariate; however, the obese group with each covariate had a nonsignificant risk of OAG compared with the non-obese group with each covariate. There was a similar tendency in most of the stratified analyses of covariates in the LHID2000, but the analyses of hypertension and hyperlipidemia were inconsistent between the LHID2000 and LHID2005. There was no adjusted hazard ratio in a few subgroups (obesity with OSA in the LHID2005, and obesity with hypotension in both the LHID2000 and LHID2005). LHID = longitudinal health insurance database; $\mathrm{OAG}=$ open-angle glaucoma; OSA = obstructive sleep apnea; $\mathrm{OPH}=$ ophthalmology. 


\section{Discussion}

In this two-database, matched-cohort study, obese adults had a higher cumulative incidence of OAG at the 13-year follow-up. Overall, obesity was a significant hazard of OAG after adjustment for multivariable covariates. Moreover, young obese adults had a remarkably higher risk of OAG than young non-obese adults.

The cardinal pathogenesis of OAG is the progressive degeneration of RGCs. Several mechanisms could be considered as to how obesity contributes to the damage of RGCs in OAG. Hormonal disequilibrium in obesity plays an integral role in the progressive impairment of RGCs. Decreased adiponectin and increased leptin resistance in obesity result in insulin resistance, dysfunctional lipid metabolism, atherosclerosis, and the activation of proinflammatory cytokines and oxidative stress, which lead to vascular hypo-perfusion and chronic inflammation of RGCs [18-20]. OAG is firmly associated with neurodegenerative pathogeneses such as dysregulation of the brain-derived neurotrophic factor (BDNF), phosphorylation of the tau protein, and overexpression of the apolipoprotein E (APOE) gene [21-23]. These neurodegenerative pathways have crucial connections with obesity. $\mathrm{BDNF}$ is a potential neuroprotective molecule that prevents neuron damage and synaptic disturbances. Recent studies have demonstrated that decreased BDNF has a profound effect on neurodegenerative diseases in the obese population [24]. Furthermore, leptin resistance in obesity plays a critical role in the formation of tau phosphorylation, which may have a detrimental effect on the impairment of RGCs [25]. Meanwhile, the fat-mass and obesity-associated (FTO) gene, strongly related to obesity, was found to be associated with risk of Alzheimer's disease through interaction with the APOE gene [26], and modulates cholesterol metabolism in the central neural network [23]. It is possible that obesity orchestrates a similar genetic vulnerability in the pathogenesis of OAG.

This was an Asian population-based study used to substantiate the association between obesity and $\mathrm{OAG}$, and the results suggest that obesity is a potential risk factor for OAG, especially in young obese adults. This result, however, contradicts those of some previous population-based studies, in which the increase of BMI was associated with a lower risk of OAG. There are several possible explanations for this divergence. First, we only included truly obese adults, because physicians in Taiwan coded obesity or morbid obesity as patients with a BMI $\geq 30 \mathrm{~kg} / \mathrm{m}^{2}$ who intended to seek medical assistance (e.g., including dietary consultation, enrollment in a weight-loss program, the use of anti-obesity drugs, or evaluation for bariatric surgery) [27]. Likewise, Newman-Casey's study, in which the result showed obesity is a risk factor of OAG, also included obese patients as the study population [14]. By contrast, the study by Pasquale and Ramdas included patients with a wide range of BMI values, which were not thoroughly specific for an obese population [15]. In Kim's study, they adopted the overweight status (BMI $>25 \mathrm{~kg} / \mathrm{m}^{2}$ ) to evaluate the risk of OAG instead of the obese status [16]. Body fat is the critical factor that induces a series of physical dysfunctions in obesity. However, increase in BMI cannot truly reflect the increased mass of body fat, especially in adults with who are overweight or have a normal weight [28]. Further, obesity contributes to more severe morbidities and mortality than overweight [29]. Several studies have even reported that overweight is related to lower mortality rates than normal weight $[30,31]$. This is why our results showed that obesity is a significant hazard of OAG, because this study recruited truly obese adults who were in need of medical assistance.

Second, previous studies have included study populations aged $>40$ years, whereas our study recruited study populations aged $\geq 18$ years. In Table 2 , the risk of OAG was significantly higher in older adults than in young adults (aged $\leq 40$ years), which is compatible with the concept that OAG is a strong age-related neurodegenerative disease. In the age-stratified analysis, obesity was a nonsignificant hazard for OAG in older adults (aged $>40$ years) although there was an increased risk of OAG in obese adults. This result is similar to most of the previous studies in that obesity was a nonsignificant risk factor for OAG in adults aged $>40$ years. This outcome could partially determine age as the most conspicuous risk factor for OAG, and show that age-related neurodegenerative effects 
result in a more profound risk for OAG than obesity-related neurodegenerative effects. Meanwhile, the degeneration of optic neurons is susceptible to long-term chronic diseases such as hypertension and diabetes, and metabolic diseases among older obese adults. These multiple factors would make the impact of obesity inconspicuous when assessing risk of OAG.

On the other hand, our data indicated that obesity is a remarkable contributor to OAG in young adults (aged $\leq 40$ years) regardless of accumulative incidence or age-stratified analysis. This result may have suggested that without the influence of aging and longterm chronic diseases, obesity would be a decisive factor for accelerating the damage of neurons, and that obesity-related neurodegenerative effects have a crucial impact on OAG occurrence among young adults [32]. Moreover, obesity may be correlated with the pathogenesis of juvenile OAG, such as mutation of the CYP1B1 gene or the glaucomaassociated olfactomedin domain of myocilin [33,34]. With obesity trending among younger populations, it is important to raise the awareness that obesity could be a potential risk factor for OAG in young adults.

In this study, obese adults had a higher rate and frequency of ophthalmology visits than non-obese adults, which is reasonable because obese adults with diabetes, hypertension, or metabolic diseases are often asked for the ophthalmic survey of cataract, retinopathy, or macular degeneration. This may have increased the incidental diagnosis of OAG that resulted in a selection bias of higher OAG risk in obese adults. In order to reduce the effect of higher rates of ophthalmology visits among obese adults, we analyzed the risk of OAG in adults with at least one ophthalmology visit, and the data showed that obesity is still a significant risk factor for OAG after multivariable adjustment. In addition, this study showed that the obese populations skewed towards female and young adults. It is assumed that female and young adults may be more concerned with their physical stature and health than male or older adults, so that these two populations are more active in seeking medical assistance to control weight. To reduce sex and age biases, a complete matching strategy was adopted in this study to mitigate the bias of these factors.

The analysis of both databases demonstrated that men exhibit a higher risk for OAG than women, although the HR did not reach statistical significance. This phenomenon could be explained by the fact that estrogen can protect RGCs in women [35]. In the sexstratified analysis, obese men had a significantly higher HR of OAG than non-obese men. One reason may be that there are decreased adiponectin levels and an increased insulin resistance among obese men compared with non-obese men [36]. On the other hand, the risk of OAG was relatively indistinct between obese and non-obese women, which could imply that elevated circulating estrogen in obese menopausal women may reduce the risk of OAG [37].

In the multivariable analysis, diabetes mellitus showed a strong association with risk of OAG. Probable pathophysiological mechanisms include insulin resistance, vascular dysregulation, and reduction of neurotrophic factors [38]. In the covariate-stratified analysis, obesity without each covariate was shown to be a significant OAG hazard, but obesity with each covariate revealed a nonsignificant risk of OAG in the LHID2005. Most stratified analyses of covariates had a similar tendency in the LHID2000. These results indicated that even in adults without these covariates, obesity would be a prominent risk factor for OAG. Nevertheless, in adults with these covariates, mutual interaction between obesity and covariates may weaken the impact of obesity on OAG occurrence, and obesity could become a relatively inconspicuous risk factor for OAG. Another reason for the nonsignificant hazard of OAG in obese adults with covariates could be due to the small number of cases because the analysis revealed a wide confidence interval. Further, the stratified analysis of hypertension and hyperlipidemia were inconsistent between the LHID2000 and LHID2005, and a larger study population is required to elucidate their role on the association between obesity and OAG in advanced studies.

This study had several limitations. First, the data of BMI were not collected in the LHID, and the dynamic changes of weight could not be traced. Additionally, the obese 
population may have been underestimated, as most physicians code obesity only if the obese patient wants to seek out medical assistance. On the other hand, the coding of obesity is rather reliable in Taiwan, because physicians are required to code patients with obesity for medical intervention, otherwise they will face the risk of an audit and heavy penalties under the monthly review of the Bureau of National Health Insurance. In addition, the incidence rate of OAG may have been underestimated. In clinical experience, obese adults often experience a long period of obesity before coding; however, we excluded all adults diagnosed with OAG before the coding of obesity could curtail the incidence of OAG in obese adults. Second, IOP is a critical risk factor for OAG, and most studies have already reported that obesity is highly associated with elevated IOP. However, the data of IOP was not collected in the LHID, so the mutual interaction among obesity, IOP, and OAG could not be evaluated without IOP data. Third, the definition of OAG depends on the coding system of the ICD-9. To validate the accuracy of OAG, we strictly defined the outcome as (at least) a coding of OAG with a treatment involving anti-glaucoma drugs or surgeries $\geq 2$ times, adjudicated by an ophthalmologist(s) over one year. Moreover, two independent databases were used to confirm the consistency of the results. Finally, there is no personal history collected in the NHIRD, so the effect of smoking was not taken into account.

To summarize, in a Taiwanese-based population, the results of this two-database matched-cohort study suggested that obese adults have an increased hazard of OAG. In age stratification, obesity could be a potential risk factor of OAG in young adults, but obesity poses a nonsignificant risk for OAG in older adults. In this era of obesity trending among young adults, more attention should be paid to the impact of obesity on OAG occurrence, and a recommendation of ophthalmic survey should be considered in young obese adults, not only for those with metabolic diseases. A comprehensive understanding of the association between obesity and OAG could have a far-reaching influence on the lives of young obese adults. Obesity-related hormonal disequilibrium, neurologic disturbance, and genetic dysregulation are possible pathogenic mechanisms of OAG. Advanced clinical and laboratory research will be essential for elucidating the relationship between obesity and OAG.

Author Contributions: Conceptualization, W.-D.C. and Y.-H.Y.; methodology, W.-D.C., K.-L.L., C.Y.L. and Y.-H.Y.; software, C.-Y.L. and Y.-H.Y.; validation, L.-J.L., T.-J.C. and Y.-H.Y.; formal analysis, C.-Y.L. and Y.-H.Y.; investigation, W.-D.C., L.-J.L., K.-L.L., T.-J.C., C.-Y.L. and Y.-H.Y.; resources, T.-J.C. and Y.-H.Y.; data curation, W.-D.C., C.-Y.L. and Y.-H.Y.; writing-original draft preparation, W.-D.C.; writing - review and editing, L.-J.L. and Y.-H.Y. All authors have read and agreed to the published version of the manuscript.

Funding: This research received no external funding.

Institutional Review Board Statement: This study was approved by the Institutional Review Board of Chang Gung Medical Foundation (Chiayi County, Taiwan; IRB No: 201701230B0), and thoroughly adhered to the tenets of the Declaration of Helsinki.

Informed Consent Statement: Given the retrospective nature of the present study and the use of de-identified patient data in the LHID, the Institutional Review Board of Chang Gung Medical foundation approved that the requirement of informed consent was waived.

Data Availability Statement: The data presented in this study are available on request from the corresponding author. The data are not publicly available due to privacy and ethical reasons.

Acknowledgments: The authors would like to thank Health Information and Epidemiology Laboratory for the comprehensive comments and assistance in data analysis. Besides, we also would like to thank Chao-Yung Wang, the physician of the department of cardiology at Linkou Chang Gung Memorial Hospital, for the consultation of the information and epidemiology of the obese population in Taiwan.

Conflicts of Interest: The authors declare no conflict of interest. 


\section{References}

1. Hruby, A.; Hu, F.B. The Epidemiology of Obesity: A Big Picture. Pharmacoeconomics 2015, 33, 673-689. [CrossRef]

2. Finkelstein, E.A.; Khavjou, O.A.; Thompson, H.; Trogdon, J.G.; Pan, L.; Sherry, B.; Dietz, W. Obesity and severe obesity forecasts through 2030. Am. J. Prev. Med. 2012, 42, 563-570. [CrossRef]

3. Qi, L.; Cho, Y.A. Gene-environment interaction and obesity. Nutr. Rev. 2008, 66, 684-694. [CrossRef] [PubMed]

4. Heymsfield, S.B.; Wadden, T.A. Mechanisms, Pathophysiology, and Management of Obesity. N. Engl. J. Med. 2017, 376, 254-266. [CrossRef] [PubMed]

5. Pi-Sunyer, X. The medical risks of obesity. Postgrad Med. 2009, 121, 21-33. [CrossRef] [PubMed]

6. Alford, S.; Patel, D.; Perakakis, N.; Mantzoros, C.S. Obesity as a risk factor for Alzheimer's disease: Weighing the evidence. Obes Rev. 2018, 19, 269-280. [CrossRef] [PubMed]

7. Ling, C.N.Y.; Lim, S.C.; Jonas, J.B.; Sabanayagam, C. Obesity and risk of age-related eye diseases: A systematic review of prospective population-based studies. Int. J. Obes 2021, 45, 1863-1885. [CrossRef]

8. Cohen, E.; Kramer, M.; Shochat, T.; Goldberg, E.; Garty, M.; Krause, I. Relationship Between Body Mass Index and Intraocular Pressure in Men and Women: A Population-based Study. J. Glaucoma 2016, 25, e509-e513. [CrossRef]

9. Mori, K.; Ando, F.; Nomura, H.; Sato, Y.; Shimokata, H. Relationship between intraocular pressure and obesity in Japan. Int. J. Epidemiol. 2000, 29, 661-666. [CrossRef]

10. Tham, Y.C.; Cheng, C.Y. Associations between chronic systemic diseases and primary open angle glaucoma: An epidemiological perspective. Clin. Exp. Ophthalmol. 2017, 45, 24-32. [CrossRef]

11. Cheung, N.; Wong, T.Y. Obesity and eye diseases. Surv. Ophthalmol. 2007, 52, 180-195. [CrossRef] [PubMed]

12. Liu, W.; Ling, J.; Chen, Y.; Wu, Y.; Lu, P. The Association between Adiposity and the Risk of Glaucoma: A Meta-Analysis. J. Ophthalmol. 2017, 2017, 9787450. [CrossRef]

13. Springelkamp, H.; Wolfs, R.C.; Ramdas, W.D.; Hofman, A.; Vingerling, J.R.; Klaver, C.C.; Jansonius, N.M. Incidence of glaucomatous visual field loss after two decades of follow-up: The Rotterdam Study. Eur. J. Epidemiol. 2017, 32, 691-699. [CrossRef]

14. Newman-Casey, P.A.; Talwar, N.; Nan, B.; Musch, D.C.; Stein, J.D. The relationship between components of metabolic syndrome and open-angle glaucoma. Ophthalmology 2011, 118, 1318-1326. [CrossRef]

15. Pasquale, L.R.; Willett, W.C.; Rosner, B.A.; Kang, J.H. Anthropometric measures and their relation to incident primary open-angle glaucoma. Ophthalmology 2010, 117, 1521-1529. [CrossRef]

16. Kim, K.E.; Kim, M.J.; Park, K.H.; Jeoung, J.W.; Kim, S.H.; Kim, C.Y.; Kang, S.W. Epidemiologic Survey Committee of the Korean Ophthalmological, S. Prevalence, Awareness, and Risk Factors of Primary Open-Angle Glaucoma: Korea National Health and Nutrition Examination Survey 2008-2011. Ophthalmology 2016, 123, 532-541. [CrossRef]

17. Tsai, M.S.; Lee, L.A.; Tsai, Y.T.; Yang, Y.H.; Liu, C.Y.; Lin, M.H.; Hsu, C.M.; Chen, C.K.; Li, H.Y. Sleep apnea and risk of vertigo: A nationwide population-based cohort study. Laryngoscope 2018, 128, 763-768. [CrossRef] [PubMed]

18. Martin, S.S.; Qasim, A.; Reilly, M.P. Leptin resistance: A possible interface of inflammation and metabolism in obesity-related cardiovascular disease. J. Am. Coll. Cardiol. 2008, 52, 1201-1210. [CrossRef] [PubMed]

19. McArdle, M.A.; Finucane, O.M.; Connaughton, R.M.; McMorrow, A.M.; Roche, H.M. Mechanisms of obesity-induced inflammation and insulin resistance: Insights into the emerging role of nutritional strategies. Front. Endocrinol. 2013, 4, 52. [CrossRef]

20. Stewart, R.M.; Clearkin, L.G. Insulin resistance and autoregulatory dysfunction in glaucoma and retinal vein occlusion. Am. J. Ophthalmol. 2008, 145, 394-396. [CrossRef]

21. Shpak, A.A.; Guekht, A.B.; Druzhkova, T.A.; Kozlova, K.I.; Gulyaeva, N.V. Brain-Derived Neurotrophic Factor in Patients with Primary Open-Angle Glaucoma and Age-related Cataract. Curr. Eye Res. 2018, 43, 224-231. [CrossRef]

22. Chiasseu, M.; Cueva Vargas, J.L.; Destroismaisons, L.; Vande Velde, C.; Leclerc, N.; Di Polo, A. Tau Accumulation, Altered Phosphorylation, and Missorting Promote Neurodegeneration in Glaucoma. J. Neurosci. 2016, 36, 5785-5798. [CrossRef]

23. Wang, Y.; Zhou, Y.F.; Zhao, B.Y.; Gu, Z.Y.; Li, S.L. Apolipoprotein E gene epsilon4epsilon4 is associated with elevated risk of primary open angle glaucoma in Asians: A meta-analysis. BMC Med. Genet. 2014, 15, 60. [CrossRef]

24. Motamedi, S.; Karimi, I.; Jafari, F. The interrelationship of metabolic syndrome and neurodegenerative diseases with focus on brain-derived neurotrophic factor (BDNF): Kill two birds with one stone. Metab. Brain Dis. 2017, 32, 651-665. [CrossRef] [PubMed]

25. Platt, T.L.; Beckett, T.L.; Kohler, K.; Niedowicz, D.M.; Murphy, M.P. Obesity, diabetes, and leptin resistance promote tau pathology in a mouse model of disease. Neuroscience 2016, 315, 162-174. [CrossRef]

26. Keller, L.; Xu, W.; Wang, H.X.; Winblad, B.; Fratiglioni, L.; Graff, C. The obesity related gene, FTO, interacts with APOE, and is associated with Alzheimer's disease risk: A prospective cohort study. J. Alzheimers Dis. 2011, 23, 461-469. [CrossRef] [PubMed]

27. Huang, C.C.; Huang, Y.T.; Chiu, C.C. A population-based analysis of use and outcomes of laparoscopic bariatric surgery across socioeconomic groups in Taiwan. Int. J. Equity Health 2015, 14, 127. [CrossRef]

28. Nuttall, F.Q. Body Mass Index: Obesity, BMI, and Health: A Critical Review. Nutr. Today 2015, 50, 117-128. [CrossRef] [PubMed]

29. Zheng, W.; McLerran, D.F.; Rolland, B.; Zhang, X.; Inoue, M.; Matsuo, K.; He, J.; Gupta, P.C.; Ramadas, K.; Tsugane, S.; et al. Association between body-mass index and risk of death in more than 1 million Asians. N. Engl. J. Med. 2011, 364, 719-729. [CrossRef]

30. Flegal, K.M.; Kit, B.K.; Orpana, H.; Graubard, B.I. Association of all-cause mortality with overweight and obesity using standard body mass index categories: A systematic review and meta-analysis. JAMA 2013, 309, 71-82. [CrossRef] [PubMed] 
31. Orpana, H.M.; Berthelot, J.M.; Kaplan, M.S.; Feeny, D.H.; McFarland, B.; Ross, N.A. BMI and mortality: Results from a national longitudinal study of Canadian adults. Obes. Silver Spring 2010, 18, 214-218. [CrossRef]

32. Nevalainen, T.; Kananen, L.; Marttila, S.; Jylhava, J.; Mononen, N.; Kahonen, M.; Raitakari, O.T.; Hervonen, A.; Jylha, M.; Lehtimaki, T.; et al. Obesity accelerates epigenetic aging in middle-aged but not in elderly individuals. Clin. Epigenetics 2017, 9, 20. [CrossRef]

33. Li, F.; Jiang, C.; Larsen, M.C.; Bushkofsky, J.; Krausz, K.W.; Wang, T.; Jefcoate, C.R.; Gonzalez, F.J. Lipidomics reveals a link between CYP1B1 and SCD1 in promoting obesity. J. Proteome Res. 2014, 13, 2679-2687. [CrossRef]

34. Donegan, R.K.; Hill, S.E.; Freeman, D.M.; Nguyen, E.; Orwig, S.D.; Turnage, K.C.; Lieberman, R.L. Structural basis for misfolding in myocilin-associated glaucoma. Hum. Mol Genet. 2015, 24, 2111-2124. [CrossRef] [PubMed]

35. Dewundara, S.S.; Wiggs, J.L.; Sullivan, D.A.; Pasquale, L.R. Is Estrogen a Therapeutic Target for Glaucoma? Semin. Ophthalmol. 2016, 31, 140-146. [CrossRef] [PubMed]

36. Hara, T.; Fujiwara, H.; Shoji, T.; Mimura, T.; Nakao, H.; Fujimoto, S. Decreased plasma adiponectin levels in young obese males. J. Atheroscler. Thromb. 2003, 10, 234-238. [CrossRef] [PubMed]

37. Cleary, M.P.; Grossmann, M.E. Minireview: Obesity and breast cancer: The estrogen connection. Endocrinology 2009, 150, 2537-2542. [CrossRef]

38. Song, B.J.; Aiello, L.P.; Pasquale, L.R. Presence and Risk Factors for Glaucoma in Patients with Diabetes. Curr. Diab. Rep. 2016, 16, 124. [CrossRef] 